\title{
Myelin modifications after chronic sleep loss in adolescent mice
}

\author{
Michele Bellesi ${ }^{1,2, *, \dagger}$, John Douglas Haswell ${ }^{1, \dagger}$, Luisa de Vivo ${ }^{1}$, \\ William Marshall ${ }^{1}$, Patrick H Roseboom ${ }^{1}$, Giulio Tononi ${ }^{1}$ and Chiara Cirelli ${ }^{1, *}$
}

${ }^{1}$ Department of Psychiatry, University of Wisconsin-Madison, Madison, WI and ${ }^{2}$ Department of Experimental and Clinical Medicine, Section of Neuroscience and Cell Biology, Università Politecnica delle Marche, Ancona, Italy

${ }^{\dagger}$ Co-first authors

${ }^{*}$ Corresponding author. Chiara Cirelli, Department of Psychiatry, University of Wisconsin-Madison 6001 Research Park Blvd Madison, WI 53719. Email: ccirelli@wisc.edu or Michele Bellesi, Department of Experimental and Clinical Medicine, Section of Neuroscience and Cell Biology, Università Politecnica delle Marche, Via Tronto 10/A, 60126, Ancona, Italy. Email: michele.bellesi@gmail.com.

\begin{abstract}
Study Objectives: Previous studies found that sleep loss can suppress the expression of genes implicated in myelination and can have adverse effects on oligodendrocyte precursor cells. On the other hand, sleep may favor myelination by promoting the expression of genes involved in its formation and maintenance. Albeit limited, these results suggest that sleep loss can have detrimental effects on the formation and maintenance of myelin.
\end{abstract}

Methods: Here, we tested this hypothesis by evaluating ultrastructural modifications of myelin in two brain regions (corpus callosum and lateral olfactory tract) of mice exposed to different periods of sleep loss, from a few hours of sleep deprivation to 5 days of chronic sleep restriction. In addition, we measured the internodal length-the distance between consecutive nodes of Ranvier along the axon-and plasma corticosterone levels.

Results: We find that g-ratio - the ratio of the diameter of the axon itself to the outer diameter of the myelinated fiberincreases after chronic sleep loss. This effect is mediated by a reduction in myelin thickness and is not associated with changes in the internodal length. Relative to sleep, plasma corticosterone levels increase after acute sleep deprivation, but show only a trend to increase after chronic sleep loss.

Conclusions: Chronic sleep loss may negatively affect myelin.

\section{Statement of Significance}

In both rodents and humans, there is indirect evidence that sleep loss may be detrimental for myelin. The present study is the first to assess morphologically whether different regimes of sleep loss, from few hours to several days, can affect the ultrastructure of myelin. Using electron microscopy, more than 17000 myelinated axons in two different brain regions were evaluated to obtain a comprehensive view of the effects of sleep loss on the white matter. We found that chronic sleep loss can reduce myelin thickness, an effect that may have important functional consequences given the fundamental role of myelin in optimizing the information flow throughout the brain. These results suggest that sleep may be important in maintaining a healthy myelin.

Key words: sleep; mice; myelin; forebrain; electron microscopy 


\section{Introduction}

Myelin is a substance rich in lipids and proteins that wraps around nerve fibers. It is produced by oligodendrocytes and its function is to protect and insulate the axons and enhance the transmission speed of electrical impulses [1]. Optimal speed of neural transmission is crucial for the successful integration of information across distant brain regions.

Sleep is a tightly regulated phenomenon. A sleep deficit causes a compensatory increase in the intensity and duration of sleep, whereas excessive sleep reduces sleep propensity [2]. Many lines of evidence have also shown that lack of sleep leads unavoidably to negative consequences [3]. At the cellular level, sleep deprivation increases metabolic demand leading to higher mitochondrial activity, which in turn can lead to mitochondrial stress and oxidative damage $[4,5]$. In the cerebral cortex of adolescent mice, sleep loss is associated with increased cytoplasmic area occupied by mitochondria, presence of extra-large mitochondria, signs of lysosomal activation, and deposition of lipofuscin granules [6]. Moreover, lack of sleep triggers the unfolded protein response, which can slow down the synthesis of most proteins [7]. Finally, wake is associated with overall high neuronal activity combined with lower clearance of solutes from the interstitial space [8], which can lead to higher extracellular glutamate levels $[9,10]$ and increased risk for excitotoxicity. Little is known, however, about the specific effects of sleep deprivation on myelin.

Recent transcriptomic analysis performed on oligodendrocyte-enriched samples of mouse forebrain showed that the expression of hundreds of transcripts ready to be translated into proteins changes because of sleep and wake [11]. Many genes implicated in apoptosis and cellular stress (Acin1, Bcat1, Otud7b, Nr4a1, Hip1, Irf8, Traf6, HSPE1, and HSP90aa1) were upregulated during spontaneous wake and short ( $4 \mathrm{hr}$ ) sleep deprivation relative to baseline sleep. In contrast, a number of genes involved in lipid metabolism and in particular in glycerophospholipid biosynthesis-Dgat2, Cds1, Eloul7, Chka-as well as in myelination-Opalin, Pllp, Q $k$-were transcribed preferentially during sleep [11]. Opalin is thought to be important for myelin stabilization at the paranodal site [12], whereas plasmolipin participates in the biogenesis of myelin by forming membrane domains at the Golgi level [13]. Qk regulates several myelinrelated transcripts (i.e. $\mathrm{Mbp}, \mathrm{Mag}$ ) at the post-transcriptional level by modulating their mRNA stability and distribution [14]. Consequently, mice lacking $Q k$ (quaking viable mice) display a remarkable deficit of compact myelin [14].

In humans, a single night of sleep deprivation can induce widespread changes in diffusion tensor imaging (DTI) indices of white matter microstructure [15]. Moreover, a recent structural MRI study showed that white matter tracts of limbic and sensorimotor regions, mainly in the right side, displayed low values of fractional anisotropy (FA) in patients with primary insomnia [16]. Another recent structural MRI study carried out in 448 community-dwelling older adults showed that poor sleep quality was associated with reduced global FA and increased global axial diffusivity and radial diffusivity values of white matter tracts, suggesting that the white matter may be especially sensitive to sleep loss [17]. These studies, however, lack the spatial resolution to identify the exact microanatomical substrates affected by lack of sleep, leaving unanswered the question whether extended wake can have specific detrimental effects on the formation and maintenance of myelin. Experiments in animals showed, for instance, that modifications of the FA of water diffusion, which is the basis for DTI, can also be related to changes in axon diameter or axon packing density [18].

Here, we addressed this question by evaluating ultrastructural modifications of myelin in mice exposed to different regimes of sleep loss, from a few hours of sleep deprivation to $\sim 5$ days of chronic sleep restriction. We find that the ratio between the diameter of the axon itself to the outer diameter of the myelinated fiber (g-ratio) increases after chronic sleep loss, and this effect is accounted for by a decrease in myelin thickness.

\section{Materials and Methods}

\section{Animals}

Four-week-old male homozygous B6.Cg-Tg(Thy1-YFP)16Jrs/J transgenic mice were used in this study. These mice express yellow fluorescent protein (YFP) in a subset of cortical pyramidal neurons [19] and have been studied extensively to assess the link between sleep and synaptic plasticity during development [20-22]. All animal procedures followed the National Institutes of Health Guide for the Care and Use of Laboratory Animals, and facilities were reviewed and approved by the IACUC of the University of Wisconsin-Madison and were inspected and accredited by AAALAC.

\section{Experimental groups}

Mice were housed in environmentally controlled recording chambers with free access to food and water (12:12 hr light-dark cycle; lights on at 08:00). They were divided into four experimental groups (Figure 1A): sleeping (S) mice $(n=8)$ were killed during the light phase after 6-8 hr of undisturbed sleep; sleep-deprived (SD) mice $(n=6)$ were kept awake for the first $6-8 \mathrm{hr}$ of the day using novel objects; and chronically sleep-restricted (CSR) mice $(n=10)$ were sleep restricted for $4 \frac{1}{2}$ days using a combination of methods, including exposure to novel objects and gentle handling during the day and forced locomotion on a slowly rotating treadmill during the night. The treadmill $(36 \mathrm{~cm}$ long $\times 42 \mathrm{~cm}$ wide) was located above a tray filled with $2-3 \mathrm{~cm}$ of water, and the movement speed $(1.5 \mathrm{~cm} / \mathrm{s})$ was low enough that mice could easily avoid falling into the water if they moved continuously. Video cameras and/or direct visual observation were used to constantly monitor the mice while on the platform. In previous experiments, we found that this method decreases overall sleep duration by $\sim 70$ per cent [6]; recovery sleep (RS) mice $(n=3)$ were allowed to rest undisturbed for $\sim 32 \mathrm{hr}$ after CSR. In the current study, SD and CSR mice were under constant and direct visual observation by one or more experimenters. By contrast, the sleep/wake behavior of S and RS mice was assessed using motion detection monitored with infrared video cameras, to avoid disturbing the mice. As in previous studies [20, 23] behavior was quantified online at $1 \mathrm{~s}$ time resolution, thus allowing us to kill the mice after a period of well consolidated sleep ( $>45 \mathrm{~min}$, interrupted by periods of wake of $4 \mathrm{~min}$ ). All mice were killed between 14:40 and 18:00 to maintain the time of brain collection within the same $3 \mathrm{hr}$ circadian window for all experimental groups (Figure 1A). Subsets of animals in each 
experimental group were used for electron microscopy and/or confocal microscopy experiments (see below).

\section{Electron microscopy experiments}

S $(n=4), \operatorname{SD}(n=2), \operatorname{CSR}(n=3)$, and RS $(n=3)$ mice were anesthetized with isoflurane ( 3 per cent volume to oxygen), then transcardially perfused for $5 \mathrm{~s}$ with phosphate buffer $\left(\mathrm{pH} 7.4,41^{\circ} \mathrm{C}\right)$ followed by 10 min of 2.5 per cent glutaraldehyde and 4 per cent paraformaldehyde (PFA) dissolved in $0.1 \mathrm{M}$ sodium cacodylate buffer $\left(\mathrm{pH} 7.4,41^{\circ} \mathrm{C}\right)$. The percentage of time spent asleep in the last $6 \mathrm{hr}$ before the perfusion was $75.5 \pm 3.5$ per cent in $\mathrm{S}$ mice, and $64.1 \pm 7.8$ per cent in RS mice. Brains were then collected and post-fixed in the same fixative solution overnight at $4^{\circ} \mathrm{C}$. It is worth noting that although fixation has strong impact on ultrastructure, all the mice received similar amount of fixative and all samples were processed in parallel. Therefore, it is highly unlikely that the fixation and staining procedure biased the results. Coronal $120 \mu \mathrm{m}$ sections were sectioned using a vibratome and then stored in cryoprotectant solution at $-20^{\circ} \mathrm{C}$. Sections at the level of the decussation of the Anterior Commissure $(+0.14 \mathrm{~mm}$
A/P from Bregma) were used. Two circular samples $\sim 1 \mathrm{~mm}$ in diameter were punched out from the sections for each animal. The first sample was aimed at the corpus callosum (CC) superficial to the lateral ventricle (Figure 1B-D). This cut was positioned so that the sample included, in the dorso-ventral direction, both the grey matter above the cingulum of the CC and the lateral ventricle beneath the CC. Mediolaterally, it spanned the entirety of the lateral ventricle, including the lateral septal nucleus and the caudate-putamen on its borders. The second sample was positioned to include the entirety of the lateral olfactory tract (LOT; Figure 1E-G). All staining was performed at room temperature (RT) with agitation unless otherwise stated. Sections were first rinsed in cacodylate buffer and stained in 1 per cent potassium ferrocyanide/2 per cent osmium tetroxide for $1 \mathrm{hr}$ on ice and in the dark, with no agitation. Next, sections were rinsed in cacodylate buffer, stained in 1 per cent thiocarbohydrazide for 20 min, rinsed in $\mathrm{H}_{2} \mathrm{O}$, and stained in 2 per cent osmium tetroxide for $30 \mathrm{~min}$ at RT, in the dark with no agitation. Sections were then rinsed in $\mathrm{H}_{2} \mathrm{O}$, stained with 1 per cent uranyl acetate at RT for $2 \mathrm{hr}$ with no agitation, and then overnight at $4^{\circ} \mathrm{C}$. The next day the sections were rinsed in $\mathrm{H}_{2} \mathrm{O}$ and then stained in lead aspartate for $30 \mathrm{~min}\left(\mathrm{pH} 5.5,60^{\circ} \mathrm{C}\right)$, followed by rinses in $\mathrm{H}_{2} \mathrm{O}$ and

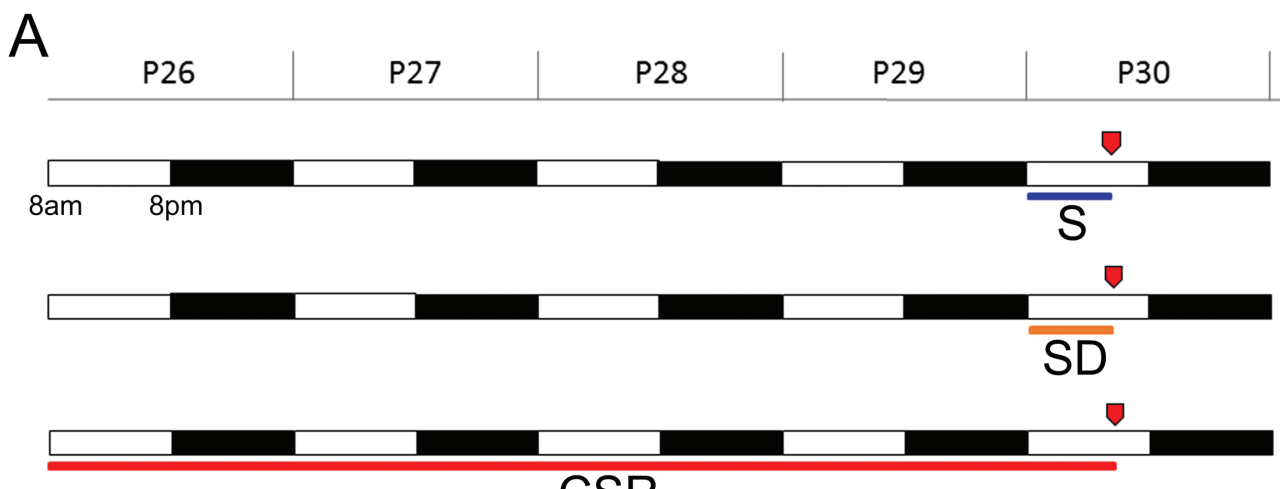

\section{CSR}
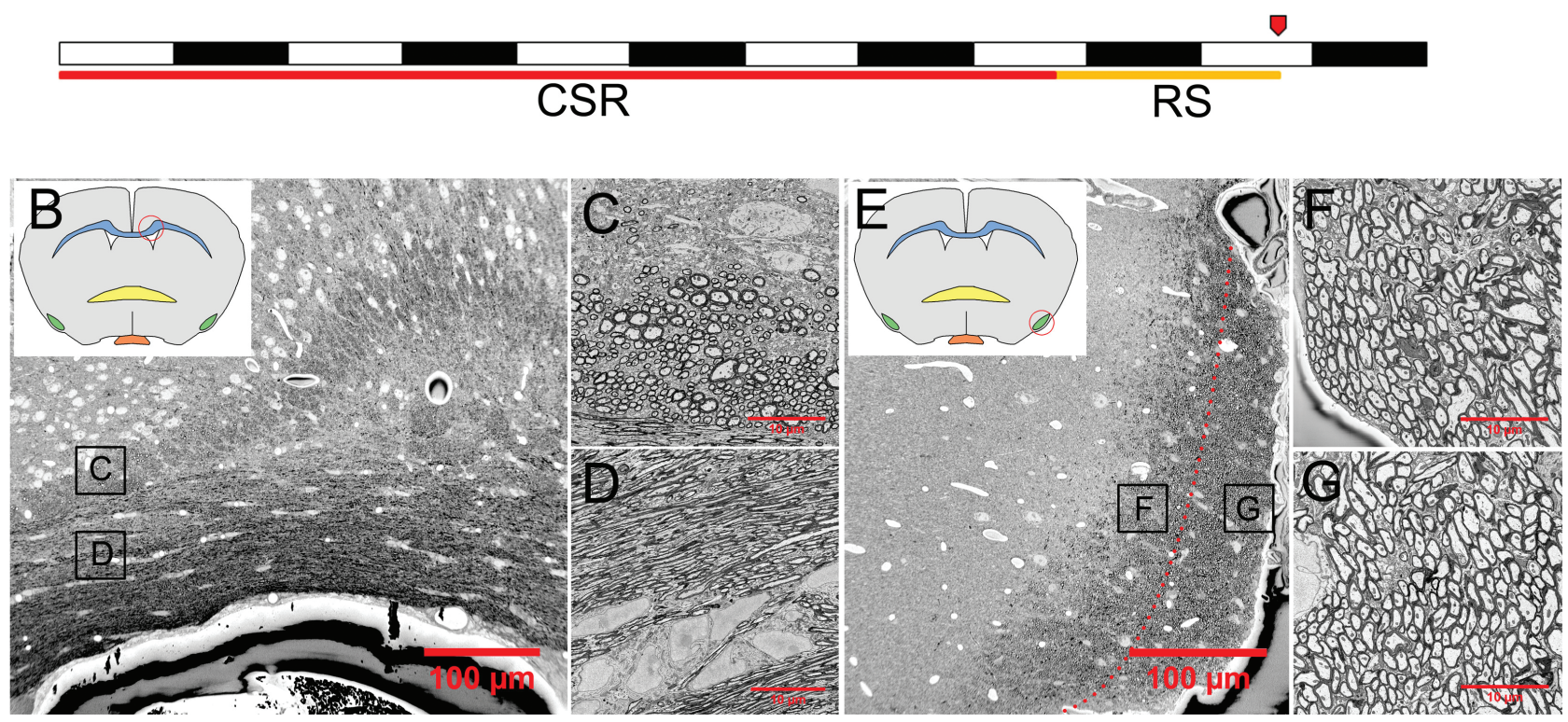

Figure 1. (A) Experimental design (P, postnatal day). S (sleep), SD (acute sleep deprivation), CSR (chronic sleep restriction), and RS (recovery after CSR). Red arrows indicate the time of perfusion. (B) Coronal section of CC. (C) and (D) Region of interest (C) selected for the analysis. Note that fibers are almost all orthogonal in this region of CC, whereas the opposite is true for the region underneath (D), where most of the fibers are parallel. (E) Section of the LOT. Medial and lateral regions are separated by a red dotted line. (F) and (G) Medial (F) and lateral (G) LOT regions of interest selected for the analysis. 
dehydration through a series of increasing alcohol concentrations ( $35,50,70,80,90,95$, and 100 per cent), and then propylene oxide. Dehydrating solutions were kept chilled in ice. Sections were infiltrated through a series of increasing concentrations of Durcupan ACM resin in propylene oxide (25, 50, and 75 per cent; $2 \mathrm{hr}$ each concentration) and then overnight in 100 per cent Durcupan. The following day, sections were moved to a newly prepared 100 per cent Durcupan solution for an additional $8 \mathrm{hr}$. Sections were then embedded in ACLAR embedding film and heated in an oven for 2 days at $60^{\circ} \mathrm{C}$. Excess ACLAR was cut away from the embedded sample under a stereomicroscope, resulting in blocks of approximately $1 \mathrm{~mm}^{2}$. The block was attached to the top surface of an aluminum pin using a conductive silver epoxy as an adhesive. The silver epoxy was then polymerized by placing the samples in a $60^{\circ} \mathrm{C}$ oven overnight. The following day, excess ACLAR and tissue were trimmed away from the sample under a stereomicroscope.

\section{Imaging}

Samples were imaged in a IIGMA VP field emission scanning electron microscope with following parameters: aperture $30 \mu \mathrm{m}$; acceleration voltage $1.8 \mathrm{kV}$; and $\sim 3 \mathrm{~nm}$ per pixel, $12000 \times 12000$ pixels per image. Within the CC immediately superficial to the lateral ventricle, the axons were horizontally oriented while above, a layer of myelinated axons oriented orthogonally to the coronal plane was present (Figure 1B-D). These orthogonally oriented myelinated axons were densely grouped on the medial portion of CC above the ventricle and were relatively sparse above the cingulum, interspersed with radially oriented unmyelinated axons. Because the medial portion had dense myelination and a visually distinct border between the white matter tract and surrounding grey matter, this region (i.e. the medial portion of CC superficial to the lateral ventricle) was selected for imaging and analysis. Contrary to the CC, the LOT sample did not have easily defined landmarks. However, at this level $(+0.14 \mathrm{~mm}$ $\mathrm{A} / \mathrm{P}$ from Bregma), the tract has the shape of a teardrop, with a wider ventromedial portion and a thinner dorsolateral portion. Although the orientation of myelinated axons varies across the LOT, with both orthogonal and obliquely oriented axons, there was no clear grouping of these differently oriented axons, and no consistent patterns across animals. Since axons on the surface of the LOT are newer, as axons are added superficially [24], we divided the tract into two sections, one located more dorsally and laterally (lateral LOT, LOTl) and the grey-matter apposing region of the LOT, located more medially (medial LOT, LOTm), both vertically spanning throughout the LOT.

\section{Image analysis}

The g-ratio is defined as the ratio of the axonal segment diameter to the total fiber segment diameter and was measured by tracing two components of the axonal fiber: the axonal segment containing the cytoplasm and membrane of the myelinated axon, and the fiber segment that includes both the axon and its surrounding myelin. The axonal segment is defined as the area contained within a selection drawn on the outer limit of the axonal membrane. The fiber segment is defined as the area contained within a selection drawn on the outer limit of the surrounding myelin. Given the dense apposition of myelinated axons, in some cases, it was difficult to determine where the outer limit of one myelin sheath ended, and the outer limit of an adjacently apposed sheath began. Thus, a straight line was assumed to bisect the two apposed sheaths from the vertices formed by their apposition. In addition to the g-ratio, the axonal segment diameter and the myelin thickness (fiber segment diameter minus axonal segment diameter)/2 were measured. Multiple images spanning an area of $\sim 4000 \mu \mathrm{m}^{2}$ were used to calculate the myelinated axon density ( $N$ of myelinated axons per $\mu \mathrm{m}^{2}$ ) for each area used for g-ratio calculation.

\section{Confocal experiments}

S $(n=4)$, SD $(n=4)$, and CSR $(n=7)$ mice were anesthetized with isoflurane (3 per cent volume to oxygen) and transcardially perfused for $30 \mathrm{~s}$ at RT with normal saline (0.9 per cent), followed by $8 \mathrm{~min}$ of $4^{\circ} \mathrm{C} 4$ per cent PFA in $0.1 \mathrm{M}$ phosphate buffer. In S mice, the percentage of time spent asleep in the last $6 \mathrm{hr}$ before the perfusion was $83.6 \pm 8.7 \%$. Brains were then extracted and postfixed in the same fixative solution overnight at $4^{\circ} \mathrm{C}$. Coronal sections $50 \mu \mathrm{m}$ thick were obtained using a vibratome and stored in $0.05 \mathrm{M}$ phosphate buffered saline (PBS) with 0.5 per cent sodium azide at $4^{\circ} \mathrm{C}$.

\section{Immunohistochemistry}

Sections (between +0.1 and $0.14 \mathrm{~mm} \mathrm{~A} / \mathrm{P}$ from Bregma) were used for immunohistochemistry. All steps were performed at RT with agitation unless otherwise stated. Sections were rinsed in $0.5 \mathrm{M}$ PBS $(3 \times 5 \mathrm{~min})$ to remove residual PFA and sodium azide and then placed in blocking solution containing 5 per cent normal goat serum, 0.3 per cent TritonX for $1.5 \mathrm{hr}$. Sections were incubated in a primary antibody solution of mouse anti-CASPR (1:1000 parts to water; abcam, ab105571) overnight without agitation, rinsed in PBS $(3 \times 5 \mathrm{~min})$, and then incubated in a secondary antibody solution of Alexa Fluor 568 goat antimouse IgG (1:500 parts to water; Invitrogen, a11004) overnight without agitation. As shown in pilot tests, the long incubation with the secondary antibody at RT was necessary to stain throughout the white matter of the $50 \mu \mathrm{m}$ thick sections. Sections were then rinsed in $0.5 \mathrm{M}$ PBS $(3 \times 5 \mathrm{~min})$, mounted to a glass slide, air dried, and secured with a coverslip with Vectashield mounting medium (H-1000, Vector). Because the structures of interest (axons within the white matter tracts) are very large and the sections are subject to possible mechanical distortion from pressure of the coverslip, varnish was built around the corners of the glass slide, to allow the coverslip to sit upon.

\section{Imaging}

Sections were imaged using a confocal microscope using PrairieView software and an UPlan FL $\mathrm{N} \times 40$ objective lens (numerical aperture 1.3). Image stacks with a resolution of $2048 \times 2048$ pixels and a $1 \mu \mathrm{m}$ stack interval were taken across the $50 \mu \mathrm{m}$ thick sections. The fields in which these image stacks were acquired were from the CC directly dorsal to the lateral ventricles. This area was selected because of its low density of Thy1-YFP+ axons, which permits a clearer attribution of CASPR+ paranodes to a single YFP+ fiber amongst densely clustered axons. Moreover, the axons within this area tend to run parallel 
to the coronal plane, which is necessary to obtain an internodal length segment in its entirety. Finally, image stacks were acquired with an $\sim 5$ per cent overlap so that internodal lengths spanning multiple fields could be reconstructed by identifying the same landmarks between adjacent fields.

\section{Image analysis}

From the image stacks previously acquired, areas where a pair of CASPR fluorescent puncta (corresponding to a pair of paranodes surrounding the node) colocalized with a Thy1-YFP+ axon were highlighted. If two of the previously highlighted areas were connected by the same axon, the internodal length between those paranodal pairs was measured in the $\mathrm{X}-\mathrm{Y}$ plane. This measurement, specifically, was taken from the internal edge of the CASPR fluorescence pair, along the Thy1-YFP axon, to the internal edge of the next CASPR fluorescence pair. The length in the Z-axis was first approximated by recording the section number in the image stack for each of the paranodes (sections were $1 \mu \mathrm{m}$ apart in the $Z$-axis), and then by using the distance between the two paranodes in the $Z$-axis and the length of the internode in the $\mathrm{X}-\mathrm{Y}$ plane to calculate the hypotenuse of those two components, to obtain the full measurement of the internode.

\section{Plasma corticosterone quantification}

S $(n=5), \operatorname{SD}(n=11), \operatorname{CSR}(n=6)$, and RS $(n=5)$ mice (all males) were used for this experiment. Some SD mice $(n=5)$ were SD for 6-8 $\mathrm{hr}$ by exposure to novel objects (SD-O) as done for the myelin experiments, whereas other animals were kept awake for 6-8 hr by gentle handling (SD-H; $n=6)$. The goal was to assess whether in our experimental conditions one type of enforced waking is more stressful than the other, since both methods were used in CSR mice. For S and RS mice, the percentage of time spent asleep in the last $6 \mathrm{hr}$ before the perfusion was $76.4 \pm 6.5 \%$ and $69.5 \pm 5.6 \%$, respectively. All mice were killed by cervical dislocation and quickly decapitated. Approximately $200 \mu \mathrm{L}$ of blood was collected from the chest cavity of each mouse, placed in a tube containing $5 \mu \mathrm{L}$ of $40 \mathrm{mg} / \mathrm{mL}$ EDTA, and centrifuged at $2000 \mathrm{~g}$ for $15 \mathrm{~min}$ at $4^{\circ} \mathrm{C}$ to separate the plasma. Plasma was assayed for corticosterone using the MP Biomedicals (Orangeburg, NY) ImmuChem corticosterone double antibody radioimmunoassay (RIA) kit. The samples were diluted 200-fold in assay diluent and all samples were assessed in duplicate in a single assay following manufacturer's instructions. According to the manufacturer, the intra-assay and inter-assay coefficients of variation (CVs) were 7.1 and 7.2 per cent, respectively.

\section{Statistical analysis}

Statistical analysis was performed using a linear mixed effects (LME) model that includes both random and fixed effects [25]. The general matrix form of the LME is

$$
y=Z u+X \beta+\epsilon
$$

where

$$
u \sim N(0, \Sigma) \text {, }
$$

and

$$
\epsilon \sim \mathrm{N}\left(0, I \sigma^{2}\right) .
$$

In this model, $\mathrm{y}$ is the vector of response variables, either g-ratio, axon diameter, or myelin thickness, $u$ is the vector of random effects (independent and normally distributed with mean zero and covariance $\Sigma$ ), and $\beta$ is the vector of fixed effects in the model. Design matrices $\mathrm{Z}$ and $\mathrm{X}$ link the response variables to the random and fixed effects, and $\epsilon$ is the residual values, assumed to be independent and normally distributed with constant variance $\sigma^{2}$.

The use of LME models offers several advantages over traditional ANOVA methods for repeated measures experiments. One advantage is the ability to handle unbalanced data, e.g. missing or corrupted observations. In the current study, the unbalanced data are due to differing numbers of axons in each sample of brain tissue. The other benefit, highly relevant in the current study, is greater flexibility and statistical power for post hoc comparisons. This increased power is due to the flexibility in the LME model to specify different covariance (random effects) structures. Having a correct variance structure allowed us to perform post hoc tests from within the model, leveraging all of the data to maximize statistical power. Repeatedmeasures ANOVA, on the other hand, can only support simple variance structure, i.e. ANOVA assumes that the variance of the differences between two levels of a factor is equal for any two levels (the sphericity assumption). This assumption is problematic, however, because even small departures from sphericity can inflate the error rate of post hoc tests [26].

To select the model structure, we first used the Akaike information criterion (AIC), identifying the set of fixed and random effects that minimize the AIC. If the fixed effect structure contained any interaction terms involving within-participant factors, we then expanded the random effects structure to also include these factors, as this is necessary to accurately test for the significance of such interactions [27]. Parameter estimation of LMEs was performed using numerical maximum likelihood estimators, implemented in R by the lmer() function of the lme4 package [28]. To test the significance of effects in the LME model, we used likelihood ratio tests. Post hoc tests were performed using the glht() function of the multcomp package in $\mathrm{R}$, with $p$-values adjusted for multiple comparisons using the singlestep method [29]. Note that when compared, the two methods (LME model, repeated-measures ANOVA) gave very similar results for the three main analyses performed in this study (the effect of condition on g-ratio, axon diameter, and myelin thickness). Specifically, the repeated-measures ANOVA analysis confirmed the primary finding of the study as reported below using LME models, i.e. a significant decrease in myelin thickness after chronic sleep restriction. Detailed results for all statistical models (LME and ANOVA) are presented in Supplementary Material.

Values of plasma corticosterone levels were compared across groups using one-way ANOVA followed by Tukey's post hoc test.

\section{Results}

\section{Myelin thinning occurs after chronic sleep loss}

We used four groups of young male mice (Figure 1A) that were either allowed to sleep ad libitum ( $>6 \mathrm{hr}$ of sleep, $\mathrm{S}$ mice) or were 
subjected to different degrees of sleep loss before brain collection: acute sleep deprivation (6-8 hr of wake enforced with novel objects, SD mice), chronic sleep restriction for $4 \frac{1}{2}$ days (CSR mice), and RS after CSR ( $4 \frac{1 / 2}{2}$ days of CSR followed by $1 \frac{112}{2}$ day of recovery sleep, RS mice). To assess the effects of sleep and wake on the white matter, we measured g-ratio, axon diameter, and myelin thickness of thousands of axons (S: $n=5918$; RS: $n=4593$; SD: $n=3045$; CSR: $n=4338$ ) in two different brain regions, the CC (Figure $1 \mathrm{~B}-\mathrm{D}$ ) and the LOT, which was divided into medial and lateral sections (LOTm, LOTl, Figure 1E-G). For each of these variables, we applied a LME model that accounts for random and fixed effects to estimate statistical significant changes among experimental conditions (see Methods). It is also worth noting that during the scoring process we never observed ultrastructural signs of demyelination for the above 17000 axons evaluated.

We first measured the g-ratio, the ratio of the inner axonal diameter to the total outer diameter, because it is a highly reliable measure of axonal myelination [30]. We found evidence of a condition effect $\left(\chi^{2}=7.83, d f=3, p\right.$-value $\left.=0.0496\right)$. Specifically, we observed that CSR mice generally had the largest g-ratio, whereas S animals generally had the lowest g-ratio, and SD/RS animals had intermediate values. Post hoc tests between conditions (corrected for multiple comparisons) confirmed these observations, revealing a significant difference between the two most "extreme" conditions, CSR and S $(z=3.205, p=0.0075,2.0$ per cent decrease), whereas RS and SD mice showed no significant differences relative to any of the other groups (Figure 2A).

For axonal diameter, we found that SD mice generally had the largest axon diameter (Figure 2B), but the effect of condition was not significant $\left(\chi^{2}=4.676, d f=3, p\right.$-value $\left.=0.1971\right)$. Conversely, for myelin thickness, we found a condition by region interaction $\left(\chi^{2}=20.149, d f=6, p\right.$-value $\left.=0.0026\right)$. Specifically, CSR mice generally had the smallest myelin thickness across brain regions with the exception of LOTl, in which myelin thickness was smallest in RS mice. There was no obvious and consistent ordering among the remaining conditions. Post hoc tests between conditions (corrected for multiple comparisons) confirmed that myelin thickness in CSR mice was significantly lower than in $S$ animals $(z=4.559, p=0.00004,7.6$ per cent decrease) and tended to be lower than in SD animals $(z=2.374, p=0.0812$, 4.6 per cent decrease), but did not differ between CSR and RS animals ( $z=1.813, p=0.2656,3.1$ per cent decrease). Myelin thickness was also significantly lower in RS animals than in S animals ( $z=2.685, p=0.0362,4.3$ per cent decrease), whereas no other significant differences were found across conditions (Figure 2C-D). To investigate whether the effect of CSR on myelin thickness varies based on axon diameter, we incorporated
A

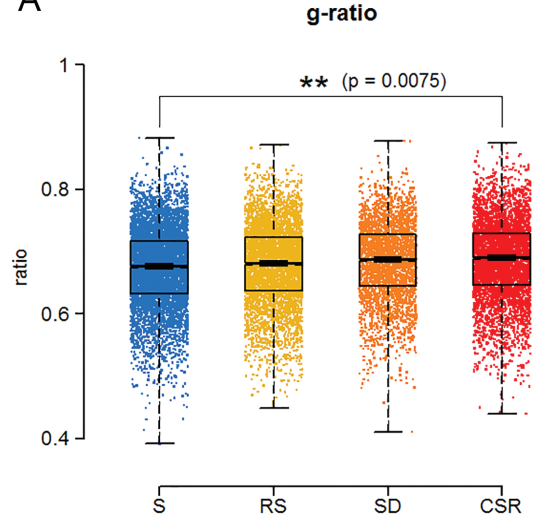

B

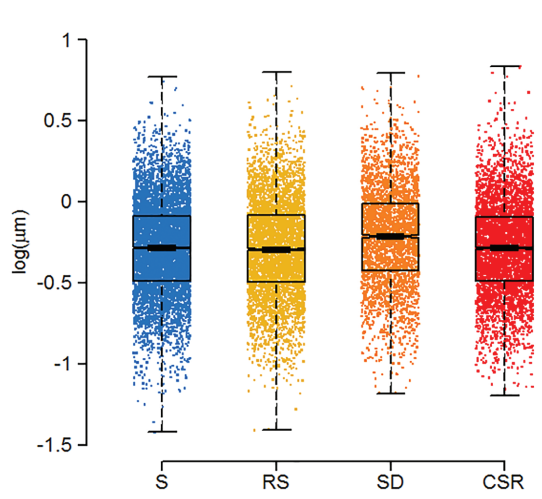

C

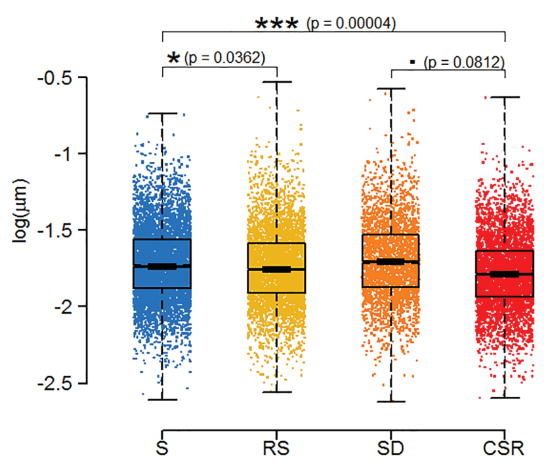

D

myelin thickness (condition by region interaction)

E

CC

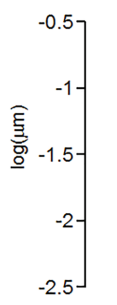

LOTV

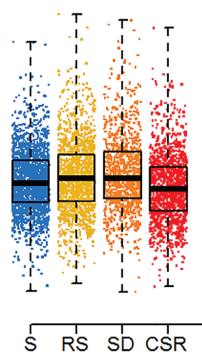

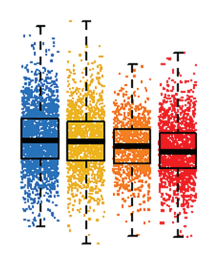

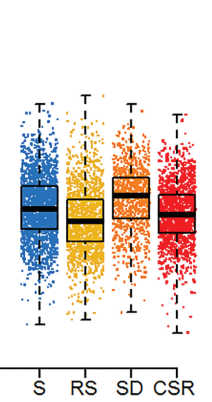

$S$ RS SD CSR

LOTd

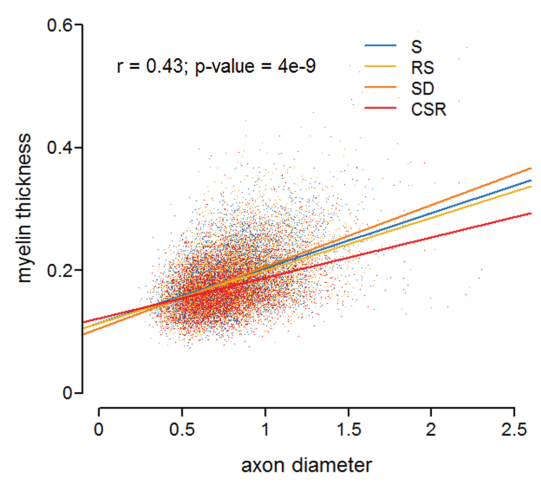

Figure 2. Myelin thinning occurs after chronic sleep loss. (A)-(C) Distribution of g-ratio (A), axon diameter (B), and myelin thickness (C) measurements for S (three mice, 5918 axons), RS (three mice, 4593 axons), SD (two mice, 3045 axons), and CSR (three mice, 4338 axons). For axon diameter and myelin thickness, a log transformation has been applied to raw values, so the units are $\log (\mu \mathrm{m})$. (D) Myelin thickness by region interaction showing that the effect of sleep loss on myelin thickness varies depending on the region. (E) Graph showing the effect of axon diameter on myelin thickness for each of the different sleep conditions, including linear regression of myelin thickness on axon diameter (bold lines) and individual observations (dots). As there was no significant interaction between condition and axon diameter, the $r$ and $p$ values refer to the regression line considering all conditions together. Note that the axes in (A), (C), and (D) are not zeroed. 

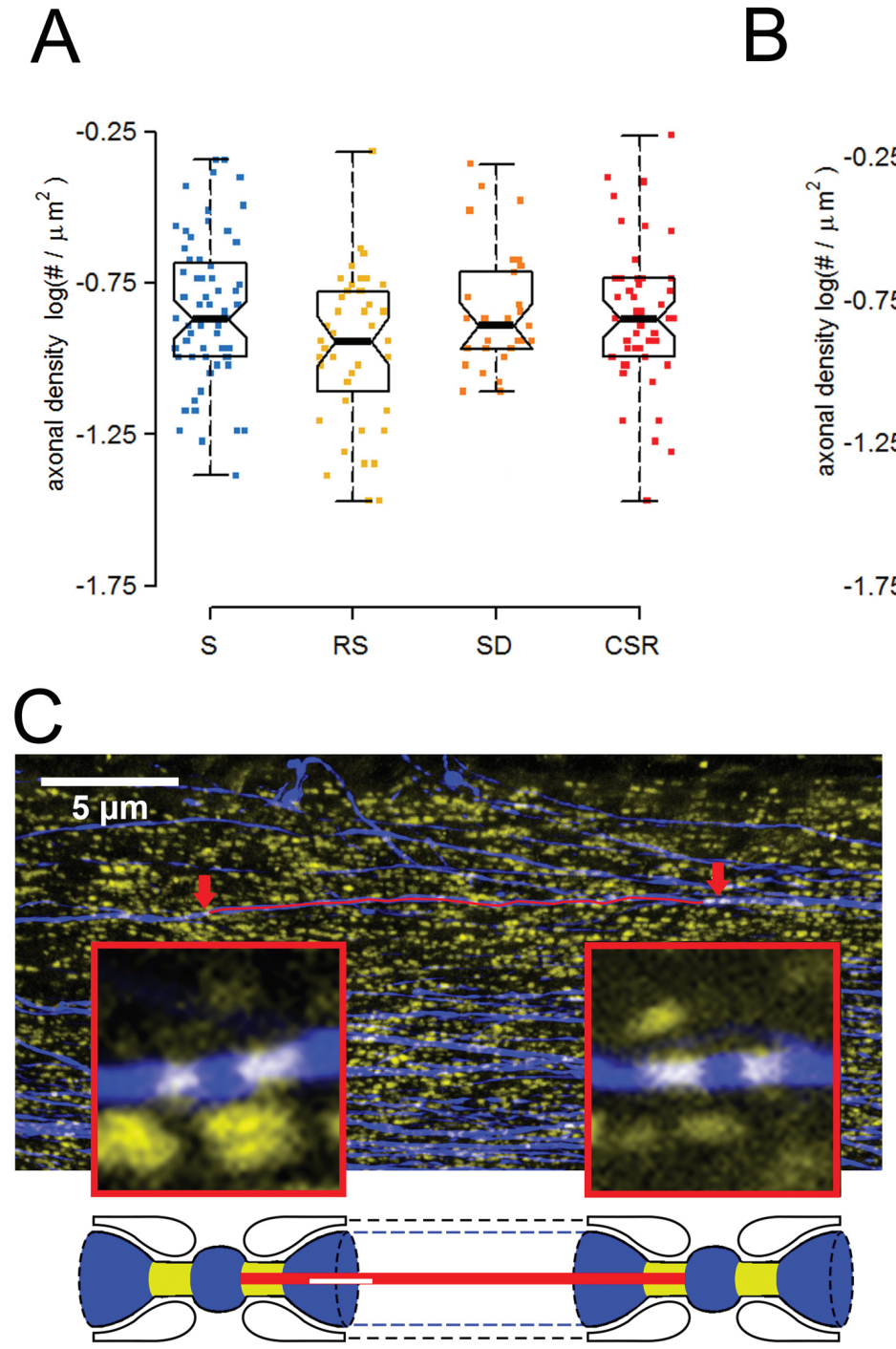
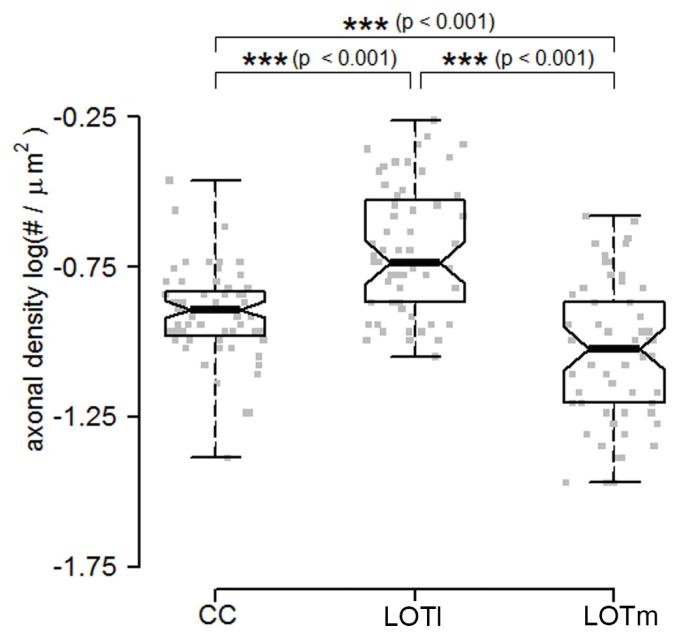

$\mathrm{D}$

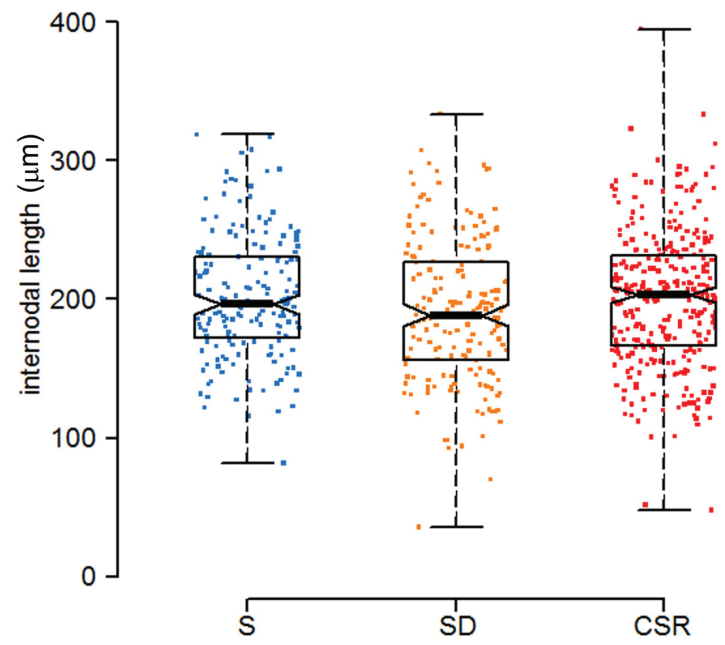

Figure 3. Sleep loss effects on density of myelinated axons and internodal length. (A) and (B) Statistical analysis showing the distribution of axonal densities (A) for S (three mice, 63 images), RS (three mice, 43 images), SD (two mice, 30 images), and CSR (three mice, 45 images) mice and the region effect (B). (C) Top: Representative CC coronal section depicting YFP+ fibers (blue) and CASPR+ puncta (yellow). Arrowheads indicate examples of nodes considered for the analysis, which are shown at larger magnification in the squared boxes. The red line along the axon indicates the internodal length. Bottom: Scheme of representative nodes of Ranvier with paranodal sites (yellow) and the red line representing the internodal length. (D) Distribution of internodal lengths for S (four mice, 173 internodes), SD (four mice, 180 internodes), and CSR (seven mice, 316 internodes) mice. Note that the axes in panels A and B are not zeroed.

a covariate (axon diameter) in the model structure, finding no significant interaction between the effect of condition and axon diameter $\left(\chi^{2}=3.799, d f=3, p\right.$-value $=0.2839$; Figure $\left.2 E\right)$.

Overall, these results indicate that the g-ratio increases after chronic sleep loss, and this result is explained by a reduction in myelin thickness rather than by an increase in axon diameter. Moreover, the effect of chronic sleep loss on myelin thickness is seen in all axons, independent of their size, and does not seem to recover with $32 \mathrm{hr}$ of RS.

\section{The density of myelinated axons is not affected by} sleep loss

Next, we quantified the density of myelinated axons in the same images used for the analysis of the g-ratio in CC and LOT regions of S, RS, SD, and CSR mice. We found no significant difference in the density of myelinated axons across the experimental conditions $\left(\chi^{2}=6.075, d f=3, p\right.$-value $\left.=0.108\right)$ (Figure $\left.3 \mathrm{~A}\right)$. However, we found that the density of myelinated axons varied across regions, being lowest in LOTm, highest in LOTl, and intermediate in CC (Figure 3B).

\section{Internodal length is not affected by sleep loss}

Myelin thickness is linearly correlated with the internodal length [31], i.e. the distance between consecutive nodes of Ranvier along the axon. Given the changes of myelin thickness in SD and S mice relative to CSR mice, we tested whether these modifications were associated with changes in internodal length. Using immunostaining against CASPR, a protein highly expressed at paranodal sites, we highlighted nodes along YFP+ axons and measured the internodal distances in a subset of 


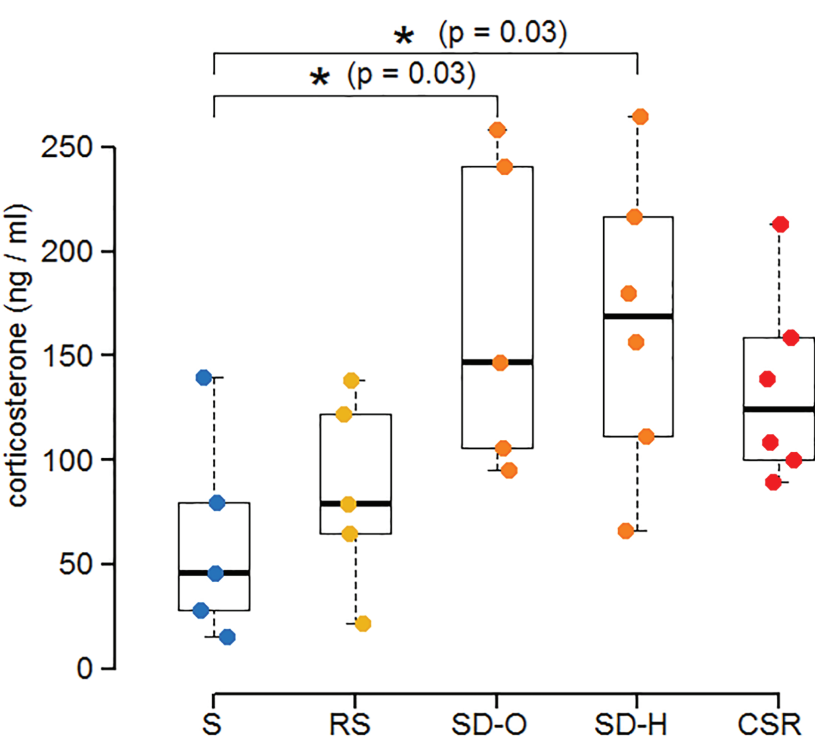

Figure 4. Plasma corticosterone levels across the experimental conditions in male mice. Acute sleep deprivation for this experiment was carried out using exposure to novel objects (SD-O) or gentle handling (SD-H).

CC axons of independent groups of S, SD, and CSR mice (S: 173 internodes; SD: 180 internodes; CSR: 316 internodes; Figure 3C). Using again an LME model, we found no evidence of a condition effect $\left(\chi^{2}=2.446, d f=2, p\right.$-value $=0.294$, Figure 3D), indicating that internodal length did not differ across experimental groups.

\section{Plasma corticosterone levels increase after sleep loss}

To determine whether the effects of sleep loss on myelin could be accounted for by increased levels of stress hormones, we measured plasma corticosterone levels in additional groups of S, SD, CSR, and RS male mice not previously used for ultrastructural experiments. SD mice included two groups: one kept awake using novel objects (SD-O) as previously done to study myelin changes and another exposed to gentle handling (SD-H), since both types of stimuli were used in CSR mice. Plasma levels of corticosterone changed across groups (ANOVA: $F_{4,22}=3.94$; $p=0.015$ ). Specifically, they were higher in both SD-O and SD-H mice relative to $S$ mice (both $p=0.03$ ), whereas CSR animals did not differ from S $(p=0.13)$, SD-O $(p=0.94)$, SD-H $(p=0.95)$, and RS mice ( $p=0.54$; Figure 4$)$. Corticosterone levels were similarly elevated with the two methods of acute sleep deprivation (SD-O vs. SD-H, $p=0.99$ ) and were comparable after baseline and RS (S vs. RS, $p=0.90$; Figure 4 ).

\section{Discussion}

We assessed the ultrastructural changes of myelinated fibers in two brain regions of adolescent mice after sleep, acute total sleep deprivation, chronic sleep restriction, and RS after chronic sleep restriction. We found that in both regions chronic sleep loss is associated with thinner myelin sheaths. This effect is not associated with changes in the number of myelinated axons and occurs in axons of all sizes. Optimal g-ratio and myelin thickness are strictly linked to conduction velocity [30, 32]. Even small deviations from the optimal values can lead to delays in the propagation of nerve pulses, affecting the frequency, propagation, and coupling of neural network oscillations, which are crucial for complex information processing [33]. Thus, these results suggest that possible detrimental effects of sleep loss on myelin cannot be ruled out at this stage.

To the best of our knowledge, this is the first structural evidence that loss of sleep can induce changes in myelin. Previously, molecular studies showed that long-term sleep deprivation down-regulates the expression of two myelin-related genes, one coding for Plasmolipin (Pllp) and the other for CD9, a membrane protein normally expressed in the mature myelin sheath [34]. More recent studies were also in line with a role of sleep in promoting myelination by showing that several genes specifically involved in myelin production and maintenance (Qk, Pllp, and Opalin) are preferably transcribed after a few hours of spontaneous sleep [11, 35]. In light of the current results, these findings suggest that loss of sleep could lead to myelin thinning by interfering with the expression of some of the genes implicated in the myelination process.

Myelin maintenance and turnover are energetically costly processes [36]. Thus, one potential mechanism to explain myelin thinning after sleep loss is that oligodendrocytes prioritize some cellular functions at the expense of others in conditions of high metabolic demands, like during periods of extended wake. Converging evidence indicates that one crucial function of oligodendrocytes is to sustain axonal energy demands, likely through mechanisms that involve oligodendrocyte glycolysis, lactate production, and shuttling to the axons [37-39]. In this respect, attention has been given to the lactate transporter MCT1, which excretes lactate outside the cell [38] and is highly expressed in oligodendrocytes [40]. Knocking-down its expression by 50 per cent caused a late-onset axonal degeneration, indicating that lactate release from oligodendrocytes is indeed crucial for axonal integrity [38]. Extracellular lactate levels are low during sleep and rise during wake [41] indicating increased demand for lactate during behavioral states that show higher metabolism. However, it is currently unknown whether extracellular lactate levels remain elevated during prolonged sleep loss, and, notably, Slc16a1, the gene coding for MCT1, is upregulated in oligodendrocyte-enriched samples of sleeping mice [11], suggesting that lactate efflux from oligodendrocytes to axons might preferably occur during sleep.

Our results suggest that the effect of sleep loss on myelin becomes morphologically visible only after several days of sleep restriction. Acute sleep deprivation for 6-8 hr did not affect myelin structure, even if molecular studies have shown that the expression of several genes involved in myelin production is reduced with a few hours of wake [11]. In addition, we found that myelin thickness does not renormalize after $\sim 32 \mathrm{hr}$ of RS, suggesting that myelin changes take time both to develop and to resolve. Recent findings show that myelin is highly plastic, as its structure is affected by changes in neuronal activity [42], learning [43], or social isolation [44]. However, it is still matter of debate whether hours, days, or longer periods are needed for myelin to remodel. Relative to controls, rats trained in the Morris water maze for 5 days showed signs of white matter modifications as detected by MRI, and elevated levels of myelin basic protein (MBP) at the histological examination [45]. Another study found increased FA, an MRI measure that evaluates the degree of restricted diffusion of protons in one orientation in the tissue, in the subcortical white matter of mice after 11 days of motor training, together with increased $\mathrm{MBP}$ expression in the same brain region [46]. However, since none 
of these studies used electron microscopy, it is difficult to say whether the increased MBP expression was due to increased myelin thickness or increased number of axons that had become myelinated. Finally, a recent study performed in both rats and humans observed white matter structural changes in the hippocampal fornix only $2 \mathrm{hr}$ after training [47], suggesting that modifications of myelin can occur more quickly than previously thought. This is in line with recent evidence showing increased FA in the white matter of human participants after 14 hours of wake [15].

In this study, chronic sleep restriction was achieved by using a combination of methods ranging from the exposure to novel objects and gentle handling during the light phase to forced locomotion during the dark phase. Previous studies using EEGimplanted mice demonstrated that this procedure can reduce total sleep time by $\sim 70$ per cent [6]. Since sleep is highly regulated, any attempt to enforce sustained wake leads to "stress," that is, to a complex physiological response to re-establish homeostasis. Thus, the stress associated with our CSR method and, in fact, with any method used to enforce sustained wake, could at least partly account for the effects on myelin. There is evidence showing that chronic stress may affect oligodendrocyte physiology. For example, 3 weeks of repeated water immersion and restraint stress in mice led to structural modifications of the oligodendrocytes in the CC, including thicker processes and increased branching of the oligodendrocytes. These morphological changes were associated with an augmented packing of the myelinated fibers as revealed by electron microscopy analysis. However, no significant changes in myelin thickness were observed following chronic stress [48]. In another study, the same stress procedure confirmed the lack of the effect on g-ratio, but found a 20 per cent reduction of the node and paranodal lengths, thus suggesting that chronic stress may alter the structural organization of the node of Ranvier [49]. Furthermore, a recent study found that 4 weeks of chronic variable stress in mice reduced the transcription of myelin-related genes (e.g. Mag, Mobp) in medial prefrontal cortex and nucleus accumbens, whereas it increased both Mog and $\mathrm{Mbp}$ expressions in the CC [50]. Collectively, these results indicate that the response of oligodendrocytes to stressful experiences is complex, region dependent, and not necessarily associated with a reduction in myelin thickness.

Classical stress responses are associated with increased levels of cortisol and/or catecholamines, and there is some indication that high levels of cortisol can have a negative impact on myelination. Administration of corticosteroids during development can delay myelination $[51,52]$, whereas early adrenalectomy increases myelination in the rat brain [53], although the evidence is not always consistent [54]. Yet, although chronic sleep loss (2 weeks) in rats consistently led to sustained activation of the sympathetic nervous system and increased release of noradrenaline thorough the brain and the body, it decreased costicosterone levels [55], and six nights of sleep restriction in humans did not affect serial $24 \mathrm{hr}$ plasma cortisol levels compared with baseline [56]. In rats, short periods of sleep restriction ( $4 \mathrm{hr}$ per day) repeated for 8 days increased plasma corticosterone levels [57], but the increase was rather small compared with the increases caused by other stressors such as restrain $[57,58]$. We found that acute sleep deprivation using exposure to novel objects significantly increased plasma corticosterone levels relative to sleep. By contrast, the levels of corticosterone in CSR mice were no longer significantly different from $\mathrm{S}$ mice, although a trend to be higher was present, and did not differ from those in SD mice, consistent with evidence that the hypothalamic-pituitary-adrenal axis of mice habituates to repeated stress $[59,60]$. On one hand, these results may suggest that it is unlikely that the effects of sleep loss on myelin can be fully accounted for by increased plasma corticosterone levels, because the temporal profile of the changes in corticosterone levels poorly correlates with the modifications in myelin content. Specifically, acute sleep deprivation robustly increased plasma corticosterone levels, but myelin thickness was not affected at this point. Moreover, at the end of CSR, plasma corticosterone levels were no longer significantly higher than after several hours of sleep, whereas myelin thickness showed a significant reduction. Finally, after $32 \mathrm{hr}$ of RS, plasma corticosterone levels did not differ from those after baseline sleep, whereas myelin thickness was still significantly smaller. On the other hand, however, it may be that even relatively mild increases in plasma corticosterone levels, when protracted, can lead to myelin changes, and the latter take time to develop and to resolve. Interestingly, the effects of high levels of noradrenaline on myelin are poorly characterized, but a few studies described a protective role of noradrenaline in experimental autoimmune encephalomyelitis, a rodent model of multiple sclerosis where myelin is severely impaired [61, 62]. Therefore, we cannot rule out that other stress-induced changes, including increased noradrenaline levels, may play a role in the myelin thinning described in the present study.

Previous studies found that axonal diameter, myelin thickness (number of lamellae), and internodal length, i.e. the distance between two consecutive nodes of Ranvier, are linearly correlated $[63,64]$ : axonal segments that show the largest diameter and the highest number of lamellae also display the longest internodal lengths. Although this linear relation does not hold very well for very small and very large fibers [31], it implies that changes in myelin thickness could lead to modifications of internodal length. However, despite changes in g-ratio and myelin thickness after chronic sleep loss, we found no change in internodal length, neither after short sleep deprivation nor after chronic sleep restriction. Thus, it is possible that internodal length changes require longer time to occur or that the small myelin change that we observed ( 8 per cent) is not enough to promote a rearrangement of node positions.

Whether sleep loss can drive myelin changes in humans remains an open question. The few studies carried out so far focused on the correlation between DTI measures and sleep loss. Although they showed changes of the FA of water diffusion in participants who experienced sleep loss, these measurements have several limitations and cannot resolve the microstructure of the white matter. By contrast, methods like positron emission spectroscopy can use myelin specific radioligands to reveal more accurately myelin changes $[65,66]$ and may in the future reveal more directly how poor sleep affects myelin in humans.

\section{Supplementary Material}

Supplementary material is available at SLEEP online.

\section{Acknowledgments}

We thank Benjamin Jones, Mattia Chini, Hirotaka Nagai, Midori Nagai, Sakiko Honjoh, Alexander V. Rodriguez, Kayla Peelman, and Giovanna Spano for helping with the chronic sleep restriction experiments. 


\section{Funding}

This study was supported by National Institutes of Health grants DP 1 OD579 (G.T.), 1R01MH091326 (G.T.), 1R01MH099231 (G.T. and C.C.), and 1P01NS083514 (G.T. and C.C.).

\section{Authors' Contributions}

M.B. devised and performed the experiments, analyzed the data, and wrote the manuscript. J.D.H. performed the experiments, analyzed the data, and wrote the manuscript. L.d.V. performed the experiments and carried out the acquisition of the EM images. P.H.R. performed corticosterone analysis. W.M. performed statistical analysis. G.T. and C.C. devised the experiments and wrote the manuscript.

\section{Notes}

Conflict of interest statement. None declared.

\section{References}

1. Baumann N, et al. Biology of oligodendrocyte and myelin in the mammalian central nervous system. Physiol Rev. 2001;81(2):871-927.

2. Borbély AA, et al. The two-process model of sleep regulation: a reappraisal. J Sleep Res. 2016;25(2):131-143.

3. Cirelli C, et al. Is sleep essential? PLoS Biol. 2008;6(8):e216.

4. Cirelli C. Cellular consequences of sleep deprivation in the brain. Sleep Med Rev. 2006;10(5):307-321.

5. Zhang J, et al. Extended wakefulness: compromised metabolics in and degeneration of locus ceruleus neurons. $J$ Neurosci. 2014;34(12):4418-4431.

6. de Vivo L, et al. Loss of sleep affects the ultrastructure of pyramidal neurons in the adolescent mouse frontal cortex. Sleep. 2016;39(4):861-874.

7. Naidoo $\mathrm{N}$, et al. Sleep deprivation induces the unfolded protein response in mouse cerebral cortex. J Neurochem. 2005;92(5):1150-1157.

8. Xie L, et al. Sleep drives metabolite clearance from the adult brain. Science. 2013;342(6156):373-377.

9. Dash MB, et al. Long-term homeostasis of extracellular glutamate in the rat cerebral cortex across sleep and waking states. J Neurosci. 2009;29(3):620-629.

10. Naylor E, et al. Simultaneous real-time measurement of EEG/EMG and L-glutamate in mice: a biosensor study of neuronal activity during sleep. J Electroanal Chem (Lausanne). 2011;656(1-2):106-113.

11. Bellesi M, et al. Effects of sleep and wake on oligodendrocytes and their precursors. J Neurosci. 2013;33(36):14288-14300.

12. Yoshikawa F, et al. Opalin, a transmembrane sialylglycoprotein located in the central nervous system myelin paranodal loop membrane. J Biol Chem. 2008;283(30):20830-20840.

13. Yaffe Y, et al. The myelin proteolipid plasmolipin forms oligomers and induces liquid-ordered membranes in the Golgi complex. J Cell Sci. 2015;128(13):2293-2302.

14. Zearfoss NR, et al. Post-transcriptional regulation of myelin formation. Biochim Biophys Acta. 2008;1779(8):486-494.

15. Elvsåshagen $\mathrm{T}$, et al. Widespread changes in white matter microstructure after a day of waking and sleep deprivation. PLoS One. 2015;10(5):e0127351.
16. Li S, et al. Reduced integrity of right lateralized white matter in patients with primary insomnia: a diffusion-tensor imaging study. Radiology. 2016;280(2):152-158.

17. Sexton CE, et al. Associations between self-reported sleep quality and white matter in community-dwelling older adults: a prospective cohort study. Hum Brain Mapp. 2017;38(11):5465-5473.

18. Fields RD. White matter in learning, cognition and psychiatric disorders. Trends Neurosci. 2008;31(7):361-370.

19. Feng $G$, et al. Imaging neuronal subsets in transgenic mice expressing multiple spectral variants of GFP. Neuron. 2000;28(1):41-51.

20. Maret S, et al. Sleep and waking modulate spine turnover in the adolescent mouse cortex. Nat Neurosci. 2011;14(11):1418-1420.

21. Nelson AB, et al. Sleep patterns and homeostatic mechanisms in adolescent mice. Brain Sci. 2013;3(1):318-343.

22. de Vivo L, et al. Developmental patterns of sleep slow wave activity and synaptic density in adolescent mice. Sleep. 2014;37(4):689-700, 700A.

23. de Vivo L, et al. Ultrastructural evidence for synaptic scaling across the wake/sleep cycle. Science. 2017;355(6324):507-510.

24. Inaki $\mathrm{K}$, et al. Laminar organization of the developing lateral olfactory tract revealed by differential expression of cell recognition molecules. J Comp Neurol. 2004;479(3):243-256.

25. Laird NM, et al. Random-effects models for longitudinal data. Biometrics. 1982;38(4):963-974.

26. Mitzel HC, et al. Circularity and multiple comparisons in repeated measure designs. $\mathrm{Br} J$ Math Stat Psychol. 1981;34(2):253-259.

27. Barr DJ. Random effects structure for testing interactions in linear mixed-effects models. Front Psychol. 2013;4:328.

28. Bates D, et al. Fitting linear mixed-effects models using lme4. J Stat Softw. 2015;67(1):1-48.

29. Bretz F, et al. Multiple Comparisons Using R. Boca Raton, FL: CRC Press; 2011.

30. Ibrahim M, et al. Relationship between myelin sheath diameter and internodal length in axons of the anterior medullary velum of the adult rat. J Neurol Sci. 1995;133(1-2):119-127.

31. Chomiak T, et al. What is the optimal value of the g-ratio for myelinated fibers in the rat CNS? A theoretical approach. PLoS One. 2009;4(11):e7754.

32. Waxman SG, et al. Relative conduction velocities of small myelinated and non-myelinated fibres in the central nervous system. Nat New Biol. 1972;238(85):217-219.

33. Pajevic $\mathrm{S}$, et al. Role of myelin plasticity in oscillations and synchrony of neuronal activity. Neuroscience. 2014;276:135-147.

34. Cirelli C, et al. Changes in brain gene expression after longterm sleep deprivation. J Neurochem. 2006;98(5):1632-1645.

35. Mongrain V, et al. Separating the contribution of glucocorticoids and wakefulness to the molecular and electrophysiological correlates of sleep homeostasis. Sleep. 2010;33(9):1147-1157.

36. Harris JJ, et al. The energetics of CNS white matter. J Neurosci. 2012;32(1):356-371.

37. Fünfschilling $\mathrm{U}$, et al. Glycolytic oligodendrocytes maintain myelin and long-term axonal integrity. Nature. 2012;485(7399):517-521.

38. Lee $\mathrm{Y}$, et al. Oligodendroglia metabolically support axons and contribute to neurodegeneration. Nature. 2012;487(7408):443-448.

39. Saab AS, et al. The role of myelin and oligodendrocytes in axonal energy metabolism. Curr Opin Neurobiol. 2013;23(6):1065-1072. 
40. Rinholm JE, et al. Regulation of oligodendrocyte development and myelination by glucose and lactate. J Neurosci. 2011;31(2):538-548.

41. Dash MB, et al. Extracellular levels of lactate, but not oxygen, reflect sleep homeostasis in the rat cerebral cortex. Sleep. 2012;35(7):909-919.

42. Gibson EM, et al. Neuronal activity promotes oligodendrogenesis and adaptive myelination in the mammalian brain. Science. 2014;344(6183):1252304.

43. McKenzie IA, et al. Motor skill learning requires active central myelination. Science. 2014;346(6207):318-322.

44. Liu J, et al. Impaired adult myelination in the prefrontal cortex of socially isolated mice. Nat Neurosci. 2012;15(12):1621-1623.

45. Blumenfeld-Katzir T, et al. Diffusion MRI of structural brain plasticity induced by a learning and memory task. PLoS One. 2011;6(6):e20678.

46. Sampaio-Baptista C, et al. Motor skill learning induces changes in white matter microstructure and myelination. J Neurosci. 2013;33(50):19499-19503.

47. Hofstetter $\mathrm{S}$, et al. Short-term learning induces white matter plasticity in the fornix. J Neurosci. 2013;33(31):12844-12850.

48. Miyata S, et al. Plasma corticosterone activates SGK1 and induces morphological changes in oligodendrocytes in corpus callosum. PLoS One. 2011;6(5):e19859.

49. Yamamoto A, et al. Association between chronic stressinduced structural abnormalities in Ranvier nodes and reduced oligodendrocyte activity in major depression. Sci Rep. 2016;6:23084.

50. Liu J, et al. Widespread transcriptional alternations in oligodendrocytes in the adult mouse brain following chronic stress. Deu Neurobiol. 2018;78(2):152-162.

51. Bohn MC, et al. Recovery of myelination in rat optic nerve after developmental retardation by cortisol. J Neurosci. 1982;2(9):1292-1298.

52. Dunlop SA, et al. Repeated prenatal corticosteroids delay myelination in the ovine central nervous system. J Matern Fetal Med. 1997;6(6):309-313.

53. Meyer JS, et al. Early adrenalectomy increases myelin content of the rat brain. Deu Brain Res. 1985;17(1-2):1-9.
54. Meyer JS, et al. Adrenalectomy in the developing rat: does it cause reduced or increased brain myelination? Dev Psychobiol. 1985;18(4):349-354.

55. Everson CA, et al. Sleep deprivation in the rat: IX. Recovery. Sleep. 1989;12(1):60-67.

56. Pejovic $\mathrm{S}$, et al. Effects of recovery sleep after one work week of mild sleep restriction on interleukin- 6 and cortisol secretion and daytime sleepiness and performance. Am J Physiol Endocrinol Metab. 2013;305(7):E890-E896.

57. Meerlo P, et al. Sleep restriction alters the hypothalamicpituitary-adrenal response to stress. J Neuroendocrinol. 2002;14(5):397-402.

58. Palchykova S, et al. Sleep deprivation impairs object recognition in mice. Neurobiol Learn Mem. 2006;85(3):263-271.

59. Grissom N, et al. Habituation to repeated stress: get used to it. Neurobiol Learn Mem. 2009;92(2):215-224.

60. Babb JA, et al. Habituation of hypothalamic-pituitary-adrenocortical axis hormones to repeated homotypic stress and subsequent heterotypic stressor exposure in male and female rats. Stress. 2014;17(3):224-234.

61. Polak PE, et al. Locus coeruleus damage and noradrenaline reductions in multiple sclerosis and experimental autoimmune encephalomyelitis. Brain. 2011;134(Pt 3):665-677.

62. Simonini MV, et al. Increasing CNS noradrenaline reduces EAE severity. J Neuroimmune Pharmacol. 2010;5(2):252-259.

63. Hildebrand C, et al. Relation between myelin sheath thickness and axon size in spinal cord white matter of some vertebrate species. J Neurol Sci. 1978;38(3):421-434.

64. Murray JA, et al. The relationship between internodal length and fibre diameter in the spinal cord of the cat. J Neurol Sci. 1980;45(1):29-41.

65. de Paula Faria D, et al. PET imaging of demyelination and remyelination in the cuprizone mouse model for multiple sclerosis: a comparison between [11C]CIC and [11C]MeDAS Neuroimage. 2014;87:395-402.

66. Veronese $\mathrm{M}$, et al. Quantification of [(11)C]PIB PET for imaging myelin in the human brain: a test-retest reproducibility study in high-resolution research tomography. J Cereb Blood Flow Metab. 2015;35(11):1771-1782. 